Int. J. Electrochem. Sci., 12 (2017) $9812-9828$

International Journal of

ELECTROCHEMICAL

SCIENCE

www.electrochemsci.org

\title{
Application of Newly Quiniline-3-carbonitriles as Corrosion Inhibitors on Mild Steel in 1.0 M HCl: Electrochemical Measurements, HF and DFT/B3LYP Calculations
}

\author{
Nuha Wazzan ${ }^{1, *}$ and Saedah Al-mhyawi ${ }^{1}$ \\ ${ }^{1}$ King Abdulaziz University, Chemistry Department, Faculty of Science, P.O Box 42805 Jeddah \\ 21589, Saudi Arabia \\ *E-Mail: nwazzan@kau.edu.sa
}

doi: $10.20964 / 2017.10 .81$

Received: 12 May 2017 / Accepted: 17 August 2017 / Published: 12 September 2017

In combined experimental and theoretical studies, two new derivatives of quinolone-3-carbonitrile were tested for their anti-corrosive properties for mild steel in a $1.0 \mathrm{M} \mathrm{HCl}$ medium. The studied compounds were 4-(4-nitrophenyl)-2-oxo-1,2,5,6-tetrahydrobenzo[ $h]$ quinoline-3-carbonitrile (NPQC) and 4-(furan-3-yl)-2-oxo-1,2,5,6-tetrahydrobenzo[ $h]$ quinoline-3-carbonitrile (FQC). The inhibition efficiencies were investigated using electrochemical techniques at different inhibitor's concentrations and temperatures. This study revealed that NPQC and FQC are good inhibitors, and the inhibition efficiency order was NPQC > FQC. The correlation between their molecular reactivity and inhibition efficiency was investigated by two methods (HF and DFT/B3LYP) with two basis sets $(6-31+\mathrm{G}(\mathrm{d})$ and $6-311++\mathrm{G}(\mathrm{d}, \mathrm{p}))$ in the gas phase and aqueous solution, applying the conductor polarizable continuum model of solvation. The quantum chemical parameters of the neutral and all possible protonated forms of the two inhibitors were evaluated. Very good correlations between the experimental and theoretical data were obtained. Modeling the inhibitor-Fe complexes of the neutral and protonated forms of the inhibitors predicts that N16 is the active site for the adsorption process for both inhibitors. The calculations of the energies of inhibitor-Fe complexes and the N16-Fe bond lengths justify the superior inhibition efficiency of NPQC compared to that of FQC.

Keywords: Anti-corrosive; mild steel; Quinoline-3-carbonitriles; DFT/B3LYP calculations; Quantum chemical parameters.

\section{$\underline{\text { FULL TEXT }}$}

(C) 2017 The Authors. Published by ESG (www.electrochemsci.org). This article is an open access article distributed under the terms and conditions of the Creative Commons Attribution license (http://creativecommons.org/licenses/by/4.0/). 